Kompass

Autoimmun

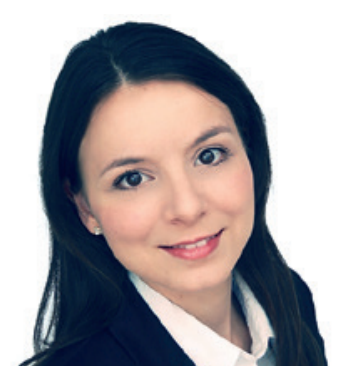

\title{
Kombinationstherapie mit Ambrisentan und Tadalafil bei CTD-PAH: Viel hilft viel?
}

\author{
Panagiota Xanthouli
}

Sektion Pulmonale Hypertonie, Thoraxklinik am Universitätsklinikum Heidelberg, Heidelberg, Deutschland

Abstract aus Kuwana M, Blair C, Takahashi T, et al.: Initial combination therapy of ambrisentan and tadalafil in connective tissue diseaseassociated pulmonary arterial hypertension (CTD-PAH) in the modified intention-to-treat population of the AMBITION study: post hoc analysis. Ann Rheum Dis 2020;79:626-634. DOI: 10.1136/annrheumdis-2019-216274

\begin{abstract}
Objectives: To evaluate initial combination therapy with ambrisentan plus tadalafil (COMB) compared with monotherapy of either agent (MONO), and the utility of baseline characteristics and risk stratification in predicting outcomes, in patients with connective tissue disease-associated pulmonary arterial hypertension (CTD-PAH) and the systemic sclerosis (SSc)-pulmonary arterial hypertension (PAH) subpopulation.
\end{abstract}

Methods: This post hoc analysis of the Ambrisentan and Tadalafil in Patients with Pulmonary Arterial Hypertension (AMBITION) study included patients with CTD-PAH from the modified intention-to-treat population. Time to clinical failure (TtCF) was assessed by baseline characteristics, treatment assignment and risk group (low, intermediate and high) at baseline and week 16. TtCF was compared between groups using Kaplan-Meier curves and Cox proportional hazards regression modelling.

Results: The analysis included 216 patients $(\mathrm{COMB}, \mathrm{n}=117$;
MONO, $n=99$ ). The risk of clinical failure was lower with $C O M B$ versus MONO (risk reduction: CTD-PAH 51.7\%, SSc-PAH 53.7\%), particularly in patients with haemodynamic parameters characteristic of typical PAH without features of left heart disease and/ or restrictive lung disease at baseline. The risk of clinical failure was lower with $\mathrm{COMB}$ versus MONO in the baseline low-risk group (HR not calculated due to no events in (OMB), baseline intermediaterisk group (HR 0.519, 95\% Cl 0.297 to 0.905) and in the week 16 low-risk group (HR 0.069, 95\% Cl 0.009 to 0.548).

Conclusions: The benefit of COMB over MONO was demonstrated in patients with CTD-PAH, particularly in those with typical $\mathrm{PAH}$ haemodynamic characteristics at baseline. COMB is appropriate for patients categorised as low risk and intermediate risk at baseline and low risk at follow-up.

Trial registration number: NCT01178073.

(c) Author(s) (or their employer(s)) 2020 


\section{Transfer in die Praxis}

\section{Hintergrund}

Die pulmonal-arterielle Hypertonie (PAH) verkompliziert die Prognose der Patienten mit Kollagenosen, vor allem der Patienten mit systemischer Sklerose (SSc), die dabei eine 3-Jahres-Überlebensrate von 56\% haben. Die AMBITION-Studie untersuchte die Wirkung der Kombinationstherapie mit Ambrisentan plus Tadalafil im Vergleich zur jeweils einzelnen Medikation als Monotherapie bei Patienten mit neu diagnostizierter PAH. Hierbei zeigten sich ein Überlebensvorteil und eine Reduktion der klinischen Ereignisse unter der Kombinationstherapie. Anhand von klinischen, laborchemischen, invasiven und nicht invasiven Parametern werden die PAH-Patienten in diverse Risikogruppen bezüglich ihrer Prognose, wie in den Risikostratifizierung-Guidelines der European Society of Cardiology (ESC)/European Respiratory Society (ERS) von 2015 empfohlen, eingeteilt.

\section{Ergebnisse der Studie}

In dieser Studie wurde die Subgruppe von Patienten mit Kollagenose-assoziierter PAH (CTD-PAH), die an der AMBITION-Studie teilnahmen, analysiert. Für die Risikoeinschätzung wurden 3, nicht invasiv gemessene Parameter von der ESC/ERS-Risikostratifizierung von Baseline verwendet: 6-Minuten-Gehstrecke, NTproBNP (N-terminal pro-B-type natriuretic peptide) und WHO-Funktionsklasse. Die Patienten wurden entsprechend in 3 Risikogruppen (niedrig, mittel, hoch) klassifiziert. Die Prognose der Patienten unter Monotherapie versus Kombinationstherapie zur Baseline und in Woche 16 wurde verglichen. Untersucht wurden die Zeit bis zur klinischen Verschlechterung und das Fortschreiten der Erkrankung anhand der genannten Risikostratifizierungsparameter. Dabei zeigte sich, dass die Patienten mit typischen CTD-PAH-Charakteristika ein besseres Ansprechen und eine bessere Prognose im Vergleich zu den
Patienten mit CTD-PAH mit kardialen oder pulmonalen Komorbiditäten haben. Die Kombinationstherapie war effektiver, vor allem für die Vermeidung der klinischen Verschlechterung bei 51,7\% der CTD-PAH-Patienten und bei 53,7\% der Patienten mit PAH bei SSC. Besonders profitiert haben Patienten mit hohem pulmonal-vaskulärem Widerstand, schlechterer Pumpfunktion, niedrigem WedgeDruck sowie normaler Lungenfunktion (d.h. TLC (Gesamt-Lungenkapazität) > 86,11\% Soll) im Vergleich zu Patienten mit hohem Wedge-Druck als Hinweis auf eine linkskardiale Vorbelastung und eine niedrige TLC. Die Kombinationstherapie verbesserte die Prognose der Patienten der mittleren Risikogruppe ab der 16. Woche im Sinne einer Prolongierung der Zeit bis zur klinischen Verschlechterung.

\section{Fazit für die Praxis}

Die therapeutische Behandlung von Patienten mit CTD-PAH soll nicht nach der Ansage «viel hilft viel», sondern nach der Risikostratifizierung und genauer Überlegung in Zentren von Expertise eingeleitet werden. Eine Kombinationstherapie verbessert die Prognose der PAH vor allem bei Patienten mit «reiner» CTD-PAH. Die kardialen und pulmonalen Komorbiditäten sind immer in Betracht zu ziehen, vor jeglichem Therapieversuch.

\section{Disclosure Statement}

Hiermit erkläre ich, dass keine Interessenskonflikte in Bezug auf den vorliegenden Kommentar bestehen.

Kontaktadresse: Dr. Panagiota Xanthouli, Sektion Pulmonale Hypertonie, Thoraxklinik am Universitätsklinikum Heidelberg, Röntgenstr. 1, 69126 Heidelberg, Deutschland, Panagiota.xanthouli@med.uni-heidelberg.de 